\title{
Chromosomal radiosensitivity of lymphocytes in South African breast cancer patients of different ethnicity: An indirect measure of cancer susceptibility
}

\author{
F Z Francies, ${ }^{1,2}$ BHSc Hons; O Herd, ${ }^{1}$ PhD; X Muller, ${ }^{1}$ MSc; A Cairns, ${ }^{3}$ MB BCh, FCS (SA); M Murdoch, ${ }^{4}$ MB BCh, FCPlastSurg (SA); \\ S Nietz, ${ }^{4} \mathrm{MB}$ BCh, FCS (SA); J P Slabbert, ${ }^{1} \mathrm{PhD}$; A Baeyens, ${ }^{1,2} \mathrm{PhD}$ \\ ${ }^{1}$ iThemba LABS/National Research Foundation, Somerset West, Western Cape, South Africa \\ ${ }^{2}$ Radiobiology, Department of Radiation Sciences, Faculty of Health Sciences, University of the Witwatersrand, Johannesburg, South Africa \\ ${ }^{3}$ Department of Surgery, Donald Gordon Medical Centre, Johannesburg, South Africa \\ ${ }^{4}$ Department of Surgery, Faculty of Health Sciences, University of the Witwatersrand, Johannesburg, South Africa
}

Correspondingauthor: A Baeyens (ans.baeyens@wits.ac.za,ansbaeyens@hotmail.com)

\begin{abstract}
Background. Breast cancer is the leading cancer among South African (SA) women. SA has citizens from diverse ethnic groups, and the lifetime risk of breast cancer differs according to ethnicity. Candidate genes for increased breast cancer risk are those involved in DNA damage repair pathways, and mutations in these genes are characterised by increased chromosomal radiosensitivity. Several European studies have shown that breast cancer patients are more sensitive to ionising radiation than healthy individuals.

Objectives. To investigate the in vitro chromosomal radiosensitivity of SA women with breast cancer and the possible influence of ethnicity and clinical parameters on chromosomal radiosensitivity.

Methods. Chromosomal radiosensitivity was analysed with the micronucleus assay using lymphocytes of breast cancer patients and healthy individuals of different ethnic groups. Lymphocytes were irradiated in vitro with $2 \mathrm{~Gy}$ or $4 \mathrm{~Gy}$, and micronuclei (MN) were scored 70 hours after irradiation. These MN frequencies were correlated with the ethnicity and clinical parameters of the breast cancer patients.

Results. MN values were higher in breast cancer patients than in healthy controls. This was noted for black and white breast cancer patients at the different radiation doses. No correlations could be demonstrated between $\mathrm{MN}$ values and clinical parameters of the breast cancer, except that $\mathrm{MN}$ values were significantly higher in oestrogen receptor (ER)-positive breast cancers.

Conclusion. SA breast cancer patients have elevated chromosomal radiosensitivity compared with healthy controls. ER positivity also influences chromosomal radiosensitivity.

S Afr Med J 2015;105(8):675-678. DOI:10.7196/SAMJnew.8266
\end{abstract}



According to South Africa (SA)'s most recent cancer registry, breast cancer is the leading cancer among SA women, with a lifetime risk of 1 in $34 .^{[1]}$ SA is a country with citizens of diverse ethnicity: black African (80.2\%), white Caucasian (8.5\%), mixed/ coloured (8.9\%) and Indian/Asian (2.4\%). ${ }^{[2]}$ The lifetime risk of breast cancer differs according to ethnicity: $1 / 52$ in black women, $1 / 22$ in coloured women, $1 / 19$ in Indian women and $1 / 18$ in white women. ${ }^{[1]}$ While the incidence is lowest among black women, it is rising as a result of increased life expectancy and urbanisation, which leads to lifestyle changes that elevate exposure to known risk factors for breast cancer such as dietary changes, decreased exercise, delayed and decreased parity, and reduction in breastfeeding. ${ }^{[3]}$ Although there is a lower incidence of breast cancer in SA in comparison with developed countries, the mortality rate of existing breast cancer patients is higher owing to limited access to diagnostic centres, particularly in rural areas, lack of awareness, low standards of healthcare facilities and limited screening. ${ }^{[4]}$

Familial breast cancer caused by mutations in high-penetrance genes such as BRCA 1 and BRCA 2 accounts for only $5 \%$ of all breast cancers. The majority of breast cancers are sporadic and are due to mutations in a number of low-penetrance genes. Candidate genes for breast cancer risk include those involved in DNA damage repair pathways. Mutations in genes regulating these pathways are characterised by increased chromosomal radiosensitivity. ${ }^{[5]}$ Measurement of chromosomal radiosensitivity has been used as an indirect measure of cancer susceptibility. The association between chromosomal radiosensitivity and cancer risk is supported by the following facts: cancer-prone disorders such as ataxia telangiectasia present with high chromosomal radiosensitivity; elevated chromosomal radiosensitivity is an indicator of defects in DNA repair that could lead to the chromosomal instability often observed in cancer; and chromosomal radiosensitivity is linked with early events in carcinogenesis. ${ }^{[6]}$

Several studies (reviewed in Cardinale et al. ${ }^{[7]}$ ) on European, Asian and American populations have shown breast cancer patients to have elevated chromosomal radiosensitivity compared with healthy individuals. Studies on the chromosomal radiosensitivity of breast cancer patients have never been performed in SA.

Chromosomal radiosensitivity can be measured using the cytokinesis-block micronucleus assay. Micronuclei (MN) are small nuclei that form in the cytoplasm when chromosomes or chromosome fragments are not incorporated into the daughter nuclei subsequent to cell division. MN can contain whole chromosomes (mis-segregated during mitosis) or acentric fragments, which are usually the result of misrepaired or unrepaired DNA double-strand breaks. $\mathrm{MN}$ are counted in cells that have undergone a single division; however, cytokinesis is blocked by adding cytochalasin B, which results in binucleated (BN) cells. This assay is well established, robust and can be performed on lymphocytes, which are easily obtained through venepuncture. The automation of MN scoring with the Metafer 4 platform (MetaSystems, Germany) has minimised the variability of the assay and rendered it rapid and less subjective. 


\section{Objective}

To measure the chromosomal radiosensitivity of SA breast cancer patients in a case-control study design with the micronucleus assay and the Metafer 4 scoring system. Recent studies have shown how tumour characteristics of breast cancer can differ among different ethnic groups. ${ }^{[8,9]}$ The differences between the ethnic groups point to differences in the underlying biology of the disease and led to the idea of comparing chromosomal radiosensitivity in different ethnic groups. We also assessed whether there was an influence of clinical parameters on chromosomal radiosensitivity.

\section{Materials and methods Subjects}

Blood samples were collected from 68 breast cancer patients (mean age (standard deviation (SD)) 52 (12)) recruited from Charlotte Maxeke Johannesburg Academic Hospital (CMJAH), a public hospital in Johannesburg, Gauteng Province, South Africa, and Donald Gordon Medical Centre, a private hospital in Johannesburg. We included 30 black breast cancer patients (mean age 47 (12) years), 25 white breast cancer patients (mean age 59 (11) years), 7 Indian breast cancer patients (mean age 45 (8) years) and 6 coloured breast cancer patients (mean age 49 (8) years). Exclusion criteria included prior chemo- and/ or radiotherapy. Clinical and biographical information on the patients was obtained through questionnaires and hospital files. All patients were categorised by race (black, white, Indian, coloured) based on patients' selfreported data from the questionnaires. Most of the breast cancer patients $(80.0 \%)$ had invasive ductal carcinomas, of which $31.1 \%$ were stage 0 - I, $53.3 \%$ stage II and $15.6 \%$ stage III. Overall $74.1 \%$ were oestrogen receptor (ER)-positive, $63.8 \%$ were progesterone receptor $(\mathrm{PG})$-positive and $78.6 \%$ were human epidermal growth factor receptor 2 (HER2)negative. No participant was HIV-positive.

Blood samples from 70 healthy controls (mean age (SD) 35 (12) years), including 20 black women (mean age 36 (15) years), 35 white women (mean age 36 (10) years), 8 Indian women (mean age 32 (6) years) and 7 coloured women (mean age 31 (13) years), were also collected. The healthy donors were staff members and students from CMJAH, where the study was undertaken. All donors signed informed consent. Ethical approval for the study was obtained through the Human Research Ethics Committee, University of the Witwatersrand, Johannesburg (M110248).

Irradiations and micronucleus assay The protocol for the micronucleus assay described by Herd et al. ${ }^{[10]}$ was used in this study. In brief, $0.5 \mathrm{~mL}$ of heparinised blood was added to $4.5 \mathrm{~mL}$ of RPMI 1640 (BioWhittaker, USA) supplemented with 13\% fetal bovine serum (Gibco-Invitrogen, USA), and antibiotics $(50 \mathrm{U} / \mathrm{mL}$ penicillin and $50 \mathrm{mg} / \mathrm{mL}$ streptomycin; Gibco-Invitrogen, USA). The medium was pre-warmed to $37^{\circ} \mathrm{C}$ and gassed ( $5 \% \mathrm{CO}_{2} / 95 \%$ air). Culture flasks with blood and medium were irradiated with doses of 2 Gy or 4 Gy of X-rays using a $6 \mathrm{MV}$ photon beam from a medical linear accelerator (Siemens Healthcare, Germany). A 0 Gy dose was used as a sham-irradiated control. For each dose point, two cultures were set up. Immediately after irradiation the lymphocytes were stimulated with $100 \mu \mathrm{L}$ phytohaemagglutinin (stock solution $1 \mathrm{mg}$ / $\mathrm{mL}$; Sigma-Aldrich, USA), and 23 hours later $20 \mu \mathrm{L}$ cytochalasin B (stock solution of $1.5 \mathrm{mg} / \mathrm{mL}$; Sigma-Aldrich) was added to block cytokinesis. Cells were harvested at 70 hours after stimulation using a cold $\left(4^{\circ} \mathrm{C}\right)$ hypotonic shock with $7 \mathrm{~mL} 0.075 \mathrm{M}$ $\mathrm{KCl}$ (Merck, Germany). This was followed by fixation in methanol:acetic acid:Ringer ( $0.9 \% \mathrm{NaCl})$ solution (4:1:5) (Merck). Fixed cell suspensions were dropped on coded slides and stored at $4^{\circ} \mathrm{C}$. Slides were mounted with Vectashield containing DAPI (4,6-diamidino-2-phenylindole; Vector Laboratories, USA) before being scanned automatically with the Metafer 4 system. The classifier and scoring method was based on Herd et al. ${ }^{[10]}$ Each dose point was scored by at least two scorers. All results were normalised to an $\mathrm{MN}$ frequency in $1000 \mathrm{BN}$

Table 1. Spontaneous and radiation-induced MN values in breast cancer patients and healthy controls, according to ethnicity

\begin{tabular}{|c|c|c|c|}
\hline Group & Patients & Controls & $p$-values \\
\hline \multicolumn{4}{|l|}{ Black } \\
\hline$n$ & 30 & 20 & \\
\hline \multicolumn{4}{|c|}{ MN/1 $000 \mathrm{BN}$ cells, mean (SD) } \\
\hline 0 Gy & $14(8)^{*}$ & $10(4)$ & 0.0087 \\
\hline 2 Gy & $179(30)^{*}$ & $159(31)$ & 0.0273 \\
\hline 4 Gy & $498(91)^{*}$ & $449(66)$ & 0.0324 \\
\hline \multicolumn{4}{|l|}{ White } \\
\hline$n$ & 25 & 35 & \\
\hline \multicolumn{4}{|c|}{ MN/1 000 BN cells, mean (SD) } \\
\hline 0 Gy & $16(7)^{*}$ & $11(4)$ & 0.0083 \\
\hline 2 Gy & $172(24)^{*}$ & $158(23)$ & 0.0253 \\
\hline 4 Gy & $507(79)^{*}$ & $443(35)$ & 0.0006 \\
\hline \multicolumn{4}{|l|}{ Coloured } \\
\hline$n$ & 6 & 7 & \\
\hline \multicolumn{4}{|c|}{ MN/1 000 BN cells, mean (SD) } \\
\hline 0 Gy & $12(9)$ & $12(7)$ & 0.6043 \\
\hline 2 Gy & $193(49)$ & $169(20)$ & 0.4697 \\
\hline 4 Gy & 487 (103) & $444(51)$ & 0.5281 \\
\hline \multicolumn{4}{|l|}{ Indian } \\
\hline$n$ & 7 & 8 & \\
\hline \multicolumn{4}{|c|}{ MN/1 000 BN cells, mean (SD) } \\
\hline 0 Gy & $11(6)$ & $11(5)$ & 0.8427 \\
\hline 2 Gy & $172(28)$ & $177(34)$ & 0.8000 \\
\hline 4 Gy & $467(91)$ & $470(74)$ & 0.9305 \\
\hline \multicolumn{4}{|l|}{ All groups } \\
\hline$n$ & 68 & 70 & \\
\hline \multicolumn{4}{|c|}{ MN/1 000 BN cells, mean (SD) } \\
\hline 0 Gy & $14(7)^{*}$ & $11(5)$ & 0.0012 \\
\hline 2 Gy & $177(30)^{*}$ & $161(27)$ & 0.0014 \\
\hline 4 Gy & $497(86)^{*}$ & $448(52)$ & 0.0001 \\
\hline${ }^{*}$ Significantly di & & & \\
\hline
\end{tabular}


cells. Radiation-induced MN values were obtained by subtracting baseline ( 0 Gy dose) values from those obtained in irradiated samples.

Statistical analysis was performed with Graphpad Prism 6. Differences between means of MN yields of patients and controls in black and white populations were tested for significance with the unpaired Student's $t$-test. For comparison of differences in $\mathrm{MN}$ values in the Indian and coloured subgroups, and between ER receptor subgroups, the Mann-Whitney $U$-test was applied. This test is used for small sample sizes. To analyse the correlations between age, clinical parameters and MN values, we used Pearson's correlation coefficient. The confidence level of the statistical tests was $95 \%$, and statistical significance was set at $p<0.05$.

\section{Results}

The results obtained with the micronucleus assay on the samples of 68 breast cancer patients and 70 healthy individuals are presented in Table 1. The mean spontaneous MN yields of all the breast cancer patients were significantly higher than those of the healthy controls $(p<0.005)$. The spontaneous MN yields were significantly correlated with the age of the healthy individuals, but this correlation could not be observed in the breast cancer patient group $(p<0.005)$. To investigate whether ethnicity had an influence on chromosomal radiosensitivity, we split the breast cancer patients and the healthy controls into four subgroups (black, white, coloured and Indian). When the patients and controls were grouped according to their ethnicity, the significantly higher number of spontaneous $\mathrm{MN}$ was only seen in the black and white patients compared with the healthy individuals of the same ethnicity.

The radiation-induced $\mathrm{MN}$ yield was calculated by subtracting the spontaneous yield from the yield in the irradiated cells. For the whole group of breast cancer patients, the mean $\mathrm{MN}$ yields were significantly higher than in the whole group of healthy individuals for both 2 Gy and 4 Gy irradiations. Grouping the samples according to their ethnicity revealed significantly higher radiation-induced MN values in the black and white breast cancer patients for both 2 Gy and 4 Gy. This could not be observed in the coloured and Indian subgroups (Table 1). Histograms of radiation-induced $\mathrm{MN}$ after $4 \mathrm{~Gy}$ for the four ethnic subgroups are presented in Fig. 1. The MN distribution after 2 Gy showed similar patterns for the four subgroups (data not shown). Although no significant differences

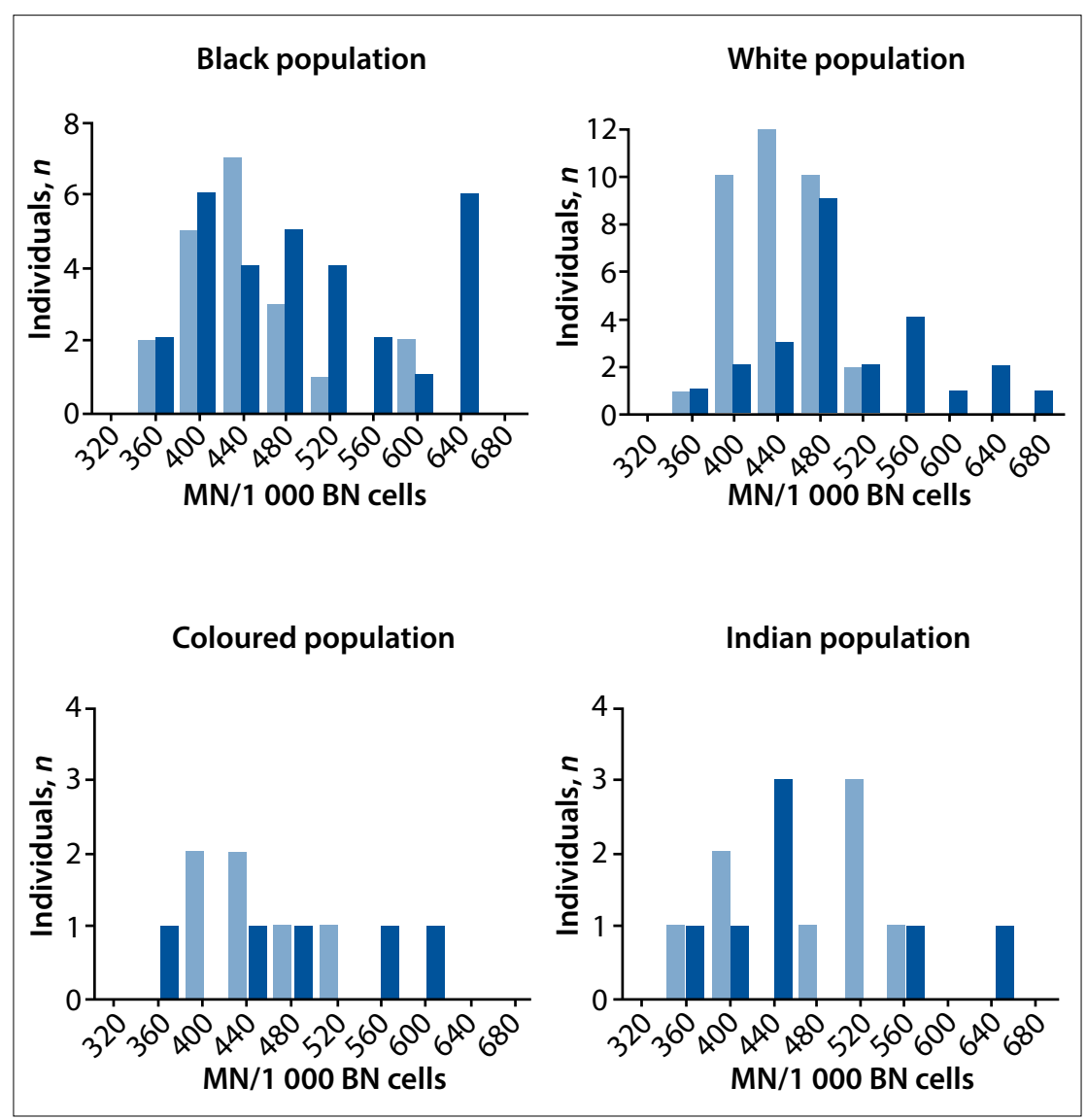

Fig. 1. Distribution of $M N$ yields after $4 \mathrm{G} y$ of breast cancer patients and healthy individuals. (Dark blue bars = breast cancer patients; light blue bars = healthy individuals.)



Fig. 2. Radiation-induced MN values of breast cancer patients grouped according to receptor status. ( ${ }^{*}$ Significantly different from the receptor-negative group.)

in mean $\mathrm{MN}$ values could be seen in the coloured group, there was a shift of values towards the higher range. This shift was not noted in the Indian population.

All the breast cancer patients were also split into groups according to clinical parameters, and $\mathrm{MN}$ values in these groups were compared. No significant correlation could be found between clinical parameters (tumour histological type, size and staging) and $\mathrm{MN}$ yields of the breast cancer patients. There was an effect of ER positivity on the MN yields (Fig. 2). Breast cancers positive for ER receptors had significantly higher radiationinduced $\mathrm{MN}$ values than ER-negative breast cancers for both $2 \mathrm{~Gy}$ and $4 \mathrm{~Gy}$ (ER-positive 
185 MN/1 000 BN cells (2 Gy), 518 MN/1 000 BN cells (4 Gy); ER-negative $159 \mathrm{MN} / 1000 \mathrm{BN}$ cells (2 Gy), $468 \mathrm{MN} / 1000 \mathrm{BN}$ cells $(4 \mathrm{~Gy}))(p=0.0031$ and $p=0.044$, respectively).

\section{Discussion}

This study investigated whether SA breast cancer patients are more sensitive than healthy individuals to DNA damage caused by ionising radiation. Since differences in breast tumour characteristics are noted between ethnic populations, we also evaluated the possible differences of chromosomal radiosensitivity in the four SA ethnic groups.

Higher spontaneous mean MN frequencies, which were seen in breast cancer patients in this study, have been linked with higher levels of genetic instability. These significantly higher mean MN values occurred mainly in the white and black patients, who had significantly higher $\mathrm{MN}$ values than controls of the same ethnicity. The higher ages of the cancer patients, which is a limitation in our study, could have played a role in the elevated spontaneous $\mathrm{MN}$ values; Thierens et al. ${ }^{[1]]}$ have suggested an increase of $0.58 \mathrm{MN} /$ year. The higher levels of spontaneous $\mathrm{MN}$ in the breast cancer patients could also suggest higher chromosomal instability, which is associated with an increased risk of cancer.

The higher chromosomal radiosensitivity observed in the whole group of SA breast cancer patients and in the white patients in this study is in agreement with several international studies. It is interesting that the significantly higher $\mathrm{MN}$ values were also seen in black breast cancer patients, who have never been studied for chromosomal radiosensitivity with the micronucleus assay. This trend is in contrast with a study performed by Wang et al. ${ }^{[12]}$ who looked at chromatid breaks in young breast cancer patients and noted significantly higher chromatid breaks in white American breast cancer patients than white controls, but not in African-American breast cancer patients compared with black controls.

The MN values of the coloured patients and controls were not significantly different, although higher $\mathrm{MN}$ values were observed in the patients. However, the small sample size of this subgroup limits conclusions. The other small subgroups were the Indian patients and controls, between whom no differences in mean $\mathrm{MN}$ values were found.

Black African breast cancer patients are known to have more aggressive tumour phenotypes than white women, and a higher prevalence of triple-negative and premenopausal breast cancers. ${ }^{[8,9]}$ These differences were not reflected in differences in chromosomal radiosensitivity of white and black breast cancer patients in our study. We found no correlation between the MN values and most of the clinical parameters investigated, which is in agreement with Baeyens et al ${ }^{[13]}$ There was an effect of ER status on MN values, with ER-positive women having significantly higher MN values. A similar trend was observed in the study of Riches et al. ${ }^{[14]}$ where patients with increased G2 radiosensitivity had a higher proportion of ER-positive tumours. The underlying reason for the higher radiation-induced $\mathrm{MN}$ in this type of breast cancer is unknown, but it could be based on interactions between the double-strand break repair kinase DNA-PK and ERs. ${ }^{[15]}$ The link between ERs and MN could suggest a prognostic value of the micronucleus assay for ER-positive breast cancers. Black and coloured women with breast cancer have a higher incidence of ER-negative cancers, ${ }^{[8,9]}$ which could have led to lower $\mathrm{MN}$ values in these groups. However, we did not observe this in our study. Enlarging the sample sizes and subgrouping the cancer patients into ethnic groups and into ER-positive and negative patients could provide greater insight.

\section{Conclusion}

Our results showed that SA breast cancer patients have elevated chromosomal radiosensitivity compared with healthy controls. The presence of ER positivity also influenced this radiosensitivity. More rigorous extended studies on the different ethnic groups are needed to validate our findings and to unravel the underlying mechanisms.

Acknowledgments. This research was supported by a grant of the South African Medical Research Council, by a 'VLIR Own Initiative Programme' between Belgium and SA (ZEIN2011PR387), and by a grant under the Nuclear Technologies in Medicine and the Biosciences Inititiative (NTeMBI), a national technology platform developed and managed by the SA Nuclear Energy Corporation and supported by the Department of Science and Technology, SA. The authors thank all the patients and healthy individuals who participated in this study.

\section{References}

1. National Health Laboratory Service. National Cancer Registry Report. Johannesburg: NHLS, 2008 2. Statistics South Africa. Mid-year population estimates. 2014. http://beta2.statssa.gov.za. (accessed 29 October 2014).

3. Porter P. 'Westernizing' women's risks? Breast cancer in lower-income countries. N Engl J Med 2008;358(3):213-216. [http://dx.doi.org/10.1056/NEJMp0708307]

4. Igene $\mathrm{H}$. Global health inequalities and breast cancer: An impending public health problen for developing countries. Breast J 2008;14(5):428-434. [http://dx.doi.org/10.1111/j.15244741.2008.00618.x]

5. Jeggo P, Lavin MF. Cellular radiosensitivity: How much better do we understand it? Int I Radiat Biol 2009;85(12):1061-1081. [http://dx.doi.org/10.3109/09553000903261263]

6. Bonassi S, Znaor A, Ceppi M, et al. An increased micronucleus frequency in peripheral blood lymphocytes predicts the risk of cancer in humans. Carcinogenesis 2007;28(3):625-626. [http://dx.doi. org/10.1093/carcin/bgll177]

7. Cardinale F, Bruzzi P, Bolognesi C. Role of micronucleus test in predicting breast cancer susceptibility: Cardinale F, Bruzzi P, Bolognesi C. Role of micronucleus test in predicting breast cancer susceptibility:
A systematic review and meta-analysis. Br J Cancer 2012;106(4):780-790. [http://dx.doi.org/10.1038/ bic.2011.567]

bjc.2011.567]
. Herd O, Francies F, Cairns A, Muller X, Slabbert JP, Baeyens A. Ethnical differences in breast cancer characteristics in South African population. Breast J 2015;21(4):447-449. [http://dx.doi.org/10.1111/ tbj.12434]

9. Dickens C, Duarte R, Zietsman A, et al. Racial comparison of receptor-defined breast cancer in Southern African women: Subtype prevalence and age-incidence analysis of nationwide cancer registry data. Cancer Epidemiol Biomarkers Prev 2014;23(11):2311-2321. [http://dx.doi.org/10.1158/10559965.EPI-14-0603]

10. Herd O, Francies F, Kotzen J, et al. Chromosomal radiosensitivity of HIV positive/negative cervical cancer patients in South Africa. Molecular Oncology Reports 2015 (in press).

1. Thierens $\mathrm{H}$, Vral A, Morthier R, Aousalah B, de Ridder L. Cytogenetic monitoring of hospital workers occupationally exposed to ionizing radiation using the micronucleus centromere assay. Mutagenesis 2000;15(3):245-249. [http://dx.doi.org/10.1093/mutagen/15.3.245]

12. Wang LE, Han CH, Xiong P, et al. Gamma-ray-induced mutagen sensitivity and risk of sporadic breast Wang LE, Han $\mathrm{CH}$, Xiong P, et al. Gamma-ray-induced mutagen sensitivity and risk of sporadic breast
cancer in young women: A case-control study. Breast Cancer Res Treat 2012;132(3):1147-1155. [http:// cancer in young women: A case-control

13. Baeyens A, Thierens $\mathrm{H}$, Claes $\mathrm{K}$, et al. Chromosomal radiosensitivity in breast cancer patients with a known or putative genetic predisposition. Br J Cancer 2002;87(12):1379-1385. [http://dx.doi. org/10.1038/sj.bjc.6600628]

14. Riches AC, Bryant PE, Steel CM, et al. Chromosomal radiosensitivity in G2-phase lymphocytes identifies breast cancer patients with distinctive tumour characteristics. Br J Cancer 2001;85(8):1157 1161. [http://dx.doi.org/10.1054/bjoc.2001.2086]

15. Medunjanin S, Weinert S, Schmeisser A, Mayer D, Braun-Dullaeus RC. Interaction of the doublestrand break repair kinase DNA-PK and estrogen receptor-alpha. Mol Biol Cell 2010;21(9):1620-1628. [http://dx.doi.org/10.1091/mbc.E09-08-0724]

Accepted 1 April 2015 